\title{
Thermal Wave Measurements with a Mirage Detection for Investigation of Thermal Diffusivity of $\mathrm{GdCa}_{4} \mathrm{O}\left(\mathrm{BO}_{3}\right)_{3}$ Single Crystals
}

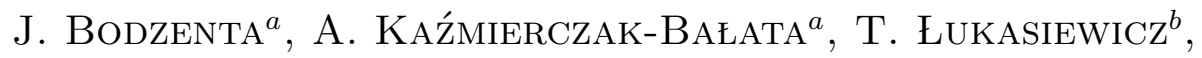 \\ B. HOFMAN ${ }^{b}$ AND J. KUCYTOWSKI ${ }^{c}$ \\ ${ }^{a}$ Institute of Physics, Silesian University of Technology \\ Krzywoustego 2, 44-100 Gliwice, Poland \\ ${ }^{b}$ Institute of Electronic Materials Technology \\ Wólczyńska 133, 01-919 Warszawa, Poland \\ ${ }^{c}$ Institute of Materials Science, University of Silesia \\ Bankowa 12, 40-007 Katowice, Poland
}

\begin{abstract}
Single crystals of $\mathrm{GdCa}_{4} \mathrm{O}\left(\mathrm{BO}_{3}\right)_{3}$ were examined to determine their thermal properties. Samples were grown by the Czochralski pulling technique. There were three types of samples: a pure crystal, the crystal doped with neodymium (4 at.\% of $\mathrm{Nd}$ ), and the third one doped with ytterbium (7 at.\% of $\mathrm{Yb}$ ). All samples were rectangular prisms with edges parallel to the axes of the optical indicatrix $X, Y, Z$ (principal axes). The thermal diffusivity was determined by means of the thermal wave method with the optical detection of the temperature disturbance based on a mirage effect. Experimental results showed anisotropy of the thermal diffusivity. The thermal diffusivity along $Y$ direction has the highest value while values obtained in $X$ and $Z$ axes are much lower. Dopants cause decrease in the thermal diffusivity for all investigated directions.
\end{abstract}

PACS numbers: 42.55.Tv, 42.70.Hj, 65.40.-b, 66.30.Xj, 66.70.-f

\section{Introduction}

Nowadays there is a great interest of producing a new non-linear-optical single crystals of high optical quality. The gadolinium calcium oxoborate $\mathrm{GdCa}_{4} \mathrm{O}\left(\mathrm{BO}_{3}\right)_{3}(\mathrm{GdCOB})$ seems to be a one of them. This crystal is widely used in laser technique as a self-frequency-doubling (SFD) laser crystal thanks 
to its excellent non-linear optical properties [1]. The rare-earth calcium oxoborate single crystals (RCOB: $\mathrm{R}=\mathrm{Pr}, \mathrm{Nd}, \mathrm{Sm}, \mathrm{Eu}, \mathrm{Dy}, \mathrm{Ho}, \mathrm{Er}$ ) are also used for surface acoustic wave (SAW) devices and as piezoelectric transducers [2]. The GdCOB crystals doped with the bismuth and lithium ions are applied as second harmonic generation (SHG) crystals. $\mathrm{Bi}^{3+}$ and $\mathrm{Li}^{+}$dopants improve the conversion efficiency in comparison with pure GdCOB single crystals [3, 4]. GdCOB has a monoclinic biaxial crystal structure and belongs to the $\mathrm{Cm}$ space group. The rare-earth dopant ions are expected to build in the lattice at gadolinium sites [5]. The unit cell parameters are: $a=8.099 \AA, b=16.031 \AA, c=3.556 \AA, \alpha=\gamma=90^{\circ}$, $\beta=101.242^{\circ}[6]$.

Application in the laser technique is determined also by thermal properties of a used crystal. The thermal expansion coefficient is an important factor during the growth process and for pure GdCOB below $900^{\circ} \mathrm{C}$ is equal to $10.5 \times 10^{-6} \mathrm{~K}^{-1}$, $8.3 \times 10^{-6} \mathrm{~K}^{-1}, 14.7 \times 10^{-6} \mathrm{~K}^{-1}$ along $X, Y, Z$ optical directions, respectively [7]. Incorporation of $\mathrm{Nd}$ dopant into GdCOB causes changes in the thermal expansion coefficient and its values for mentioned crystallographic directions in a 5.2 at.\% Nd:GdCOB crystal become equal to $11.6 \times 10^{-6} \mathrm{~K}^{-1}, 5.4 \times 10^{-6} \mathrm{~K}^{-1}$, $5.9 \times 10^{-6} \mathrm{~K}^{-1}$. A value of the specific heat influences the damage threshold of crystals especially in a pulse laser operation. For GdCOB crystal doped with 5.2 at.\% $\mathrm{Nd}$ the specific heat is about $601 \mathrm{~J} /(\mathrm{kg} \mathrm{K})$ at $300 \mathrm{~K}[8]$.

However, in case of cw lasers the damage threshold also strongly depends on the thermal conductivity (or thermal diffusivity) of a used crystal. These quantities describe heat transport properties. Low thermal conductivity results in problems with effective cooling during laser operation and may lead to unwanted effects like thermal fracture of a crystal. This can limit the maximum laser output power, degrade the quality of a laser beam and, as the result, reduce the laser efficiency.

The aim of this paper is to determine the thermal diffusivity of pure and doped GdCOB single crystals and to analyze the mechanisms of the influence on this parameter by dopants.

\section{Experimental section}

\subsection{Samples preparation}

GdCOB single crystals were grown by the Czochralski pulling technique [9] in the Institute of Technology of Electronic Materials (ITME) in Warsaw. Initial materials were prepared from boron oxides $\mathrm{B}_{2} \mathrm{O}_{3}$, gadolinium oxides $\mathrm{Gd}_{2} \mathrm{O}_{3}$ and calcium carbonate $\mathrm{CaCO}_{3}$. The mixture was kept at the temperature of $1200^{\circ} \mathrm{C}$ for $20 \mathrm{~h}$. Crystal growth proceeded from a [010] oriented seed in the iridium crucible under nitrogen atmosphere using Oxypuller 05-03 (Cyberstar). To improve the growth process an additional active heating was put at the distance of $5 \mathrm{~mm}$ over the crucible. The GdCOB compound melted congruently at $1490^{\circ} \mathrm{C}$. The crystal was rotated at $20-30 \mathrm{rpm}$. The pulling rate was $0.7-0.9 \mathrm{~mm} / \mathrm{h}$. Obtained crystals had a diameter of $35 \mathrm{~mm}$ and the length of $60 \mathrm{~mm}$. They were colorless 
TABLE I

Basic information about samples.

\begin{tabular}{c|c|c|c|c|c}
\hline \hline & Sample & Dopants & \multicolumn{3}{|c}{ Dimension $[\mathrm{mm}]$} \\
\cline { 4 - 6 } & & & {$[X]$} & {$[Y]$} & {$[Z]$} \\
\hline$\# 1$ & $\mathrm{GdCa}_{4} \mathrm{O}\left(\mathrm{BO}_{3}\right)_{3}$ & - & 9.98 & 8.16 & 9.21 \\
$\# 2$ & $\mathrm{GdCa}_{4} \mathrm{O}\left(\mathrm{BO}_{3}\right)_{3}$ & 4 at.\% of $\mathrm{Nd}$ & 10.00 & 8.32 & 9.21 \\
$\# 3$ & $\mathrm{GdCa}_{4} \mathrm{O}\left(\mathrm{BO}_{3}\right)_{3}$ & 7 at.\% of $\mathrm{Yb}$ & 9.95 & 8.36 & 9.20
\end{tabular}

and without macroscopic defects. Oriented samples were cut and polished to get rectangular prisms with faces perpendicular to the principal axes of the optical indicatrix. Basic information about samples is presented in Table I.

\subsection{Thermal properties determination}

Thermal wave methods with photodeflection signal detection are successfully used for thermal parameter measurements of volume materials as well as thin films [10]. In the case of volume materials the thermal diffusivity $\alpha$ is typically measured. The relation between the thermal diffusivity and the thermal conductivity $\kappa$ describes the equation

$$
\kappa=\rho c \alpha,
$$

where $\rho$ is the density, and $c$ is the specific heat.

The GdCOB is a low symmetry crystal and the thermal diffusivity is characterized by the second order tensor of the form

$$
\alpha_{i j}=\left[\begin{array}{ccc}
\alpha_{11} & 0 & \alpha_{31} \\
0 & \alpha_{22} & 0 \\
\alpha_{31} & 0 & \alpha_{33}
\end{array}\right],
$$

where $\alpha_{13}=\alpha_{31}$. Therefore, complete characterization of heat transport in such structure requires determination of four components of the thermal diffusivity tensor. The crystallographic system $(a, b, c)$ is not adequate to the crystallophysic system in case of monoclinic crystals. Investigated samples were cut into directions of the principal axes of optical indicatrix $(X, Y, Z)$ in which the $Y$ axis is antiparallel to the $b$ axis and the angles between the natural faces and $X$ and $Z$ axes are equal to $(X, c)=16.24^{\circ},(Z, a)=27.24^{\circ}$ [11]. For such oriented samples the thermal diffusivity tensor can be written as

$$
\alpha_{i j}=\left[\begin{array}{ccc}
\alpha_{11} & 0 & 0 \\
0 & \alpha_{22} & 0 \\
0 & 0 & \alpha_{33}
\end{array}\right] .
$$

It means that in directions of principal axes of the optical indicatrix the direction of heat flow is parallel to the temperature gradient.

The thermal diffusivity was obtained using thermal wave technique with a mirage detection. The method is based on the one-dimensional model of a 
heat propagation in the sample [12]. Let us consider a thermally thick sample. Assuming that the heat flux density through the plane $x=0$ (Fig. 1) is

$$
j(x=0)=A \cos (2 \pi f t),
$$

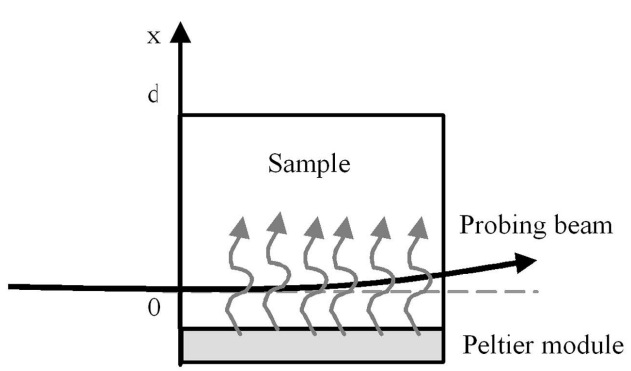

Fig. 1. Geometry of the experiment.

where $A$ is the amplitude of the heat flux, $f$ is the modulation frequency, $t$ is time, and solving Fourier-Kirchhoff equation

$$
\frac{\partial T}{\partial t}=\alpha \frac{\partial^{2} T}{\partial x^{2}},
$$

one can get the formula for the temperature field in the sample

$$
T(x, t)=T_{m} \exp \left(-\sqrt{\frac{\pi f}{\alpha}} x\right) \cos \left(2 \pi f t-\sqrt{\frac{\pi f}{\alpha}} x-\frac{\pi}{4}\right),
$$

where $T_{m}$ is the amplitude of the temperature disturbance (the thermal wave). The experimental method reported in this article bases on the investigation of the phase delay of a thermal wave propagating in the sample as a function of $\Delta x-$ distance of the probing beam from a plane $x=x_{0}$. A measured deflection signal is proportional to the temperature gradient in the sample. The phase delay of it in relation to the reference one measured at $x=x_{0}$ is given by

$$
\Delta \varphi_{s}=-\sqrt{\frac{\pi f}{\alpha}} \Delta x+\varphi_{0}
$$

where $\varphi_{0}$ is a constant. In the experiment each sample was placed on the Peltier module, which periodically heated and cooled one sample surface. An opposite surface of the module was stacked to the radiator. The temperature disturbance generated by the Peltier module propagated in the sample as a thermal wave. It caused a rise of the temperature gradient inside the crystal and connected with them gradients of the refractive index. The probing beam from the $\mathrm{He}-\mathrm{Ne}$ laser passing through the sample was deflected on the temperature disturbance existing in the crystal. Deflection of the beam was measured by a position detector. The amplitude and the phase of the signal was determined by a DSP lock-in amplifier and then collected as a measured data by the PC computer. 


\section{Results}

To determine the thermal diffusivity a phase of the signal was taken into consideration. The phase of the signal decreases with increase in the parameter $h$, which is the distance between the heater and the probing beam and is a linear function of this parameter. The thermal diffusivity was obtained from the fitting of a straight line to the data basing on Eq. (6). Exemplary results are shown in Fig. 2a. Measurements were carried out for modulation frequency of $0.4 \mathrm{~Hz}$. The thermal diffusivity determined for all investigated samples along principle axes is presented in Fig. 2b and collected in Table II.
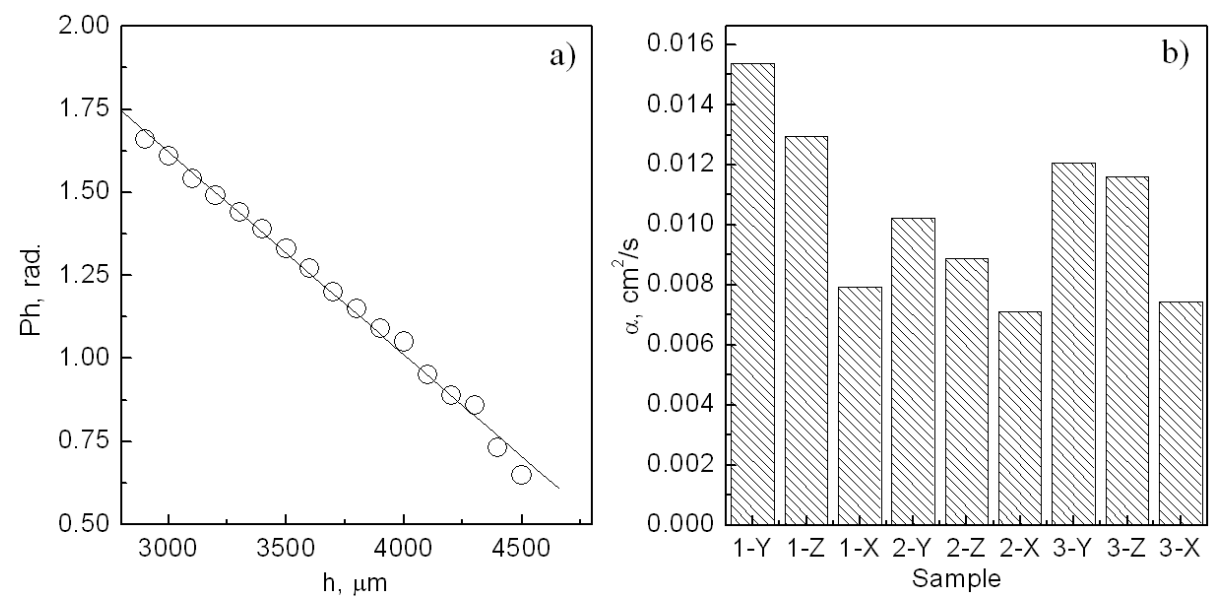

Fig. 2. The phase measured for $0.4 \mathrm{~Hz}$ modulation frequency with fitted line for 4 at\% Nd:GdCOB (a), the thermal diffusivity results obtained for all investigated samples: GdCOB (1), 4 at.\% Nd:GdCOB (2) and 7 at.\% Yb:GdCOB (3) along directions $X, Y$, $Z$ (b).

TABLE II

Thermal diffusivity results for all investigated samples.

\begin{tabular}{c|c|c|c|c|c}
\hline \hline & Sample & \multirow{2}{*}{ Dopants } & \multicolumn{3}{|c}{$\alpha \times 10^{-3}\left[\mathrm{~cm}^{2} / \mathrm{s}\right]$} \\
\cline { 4 - 6 } & & & {$[X]$} & {$[Y]$} & {$[Z]$} \\
\hline$\# 1$ & $\mathrm{GdCa} \mathrm{O}\left(\mathrm{BO}_{3}\right)_{3}$ & - & $7.92(0.61)$ & $15.38(0.66)$ & $12.96(2.07)$ \\
$\# 2$ & $\mathrm{GdCa} \mathrm{O}\left(\mathrm{BO}_{3}\right)_{3}$ & 4 at.\% of $\mathrm{Nd}$ & $7.11(1.93)$ & $10.24(0.39)$ & $8.87(1.63)$ \\
$\# 3$ & $\mathrm{GdCa}_{4} \mathrm{O}\left(\mathrm{BO}_{3}\right)_{3}$ & 7 at.\% of $\mathrm{Yb}$ & $7.44(1.09)$ & $12.08(0.26)$ & $11.62(0.57)$
\end{tabular}

The results show that the thermal diffusivity has a different value in each measurement direction. The lowest value was obtained for the $X$ direction. Values got in the $Y$ direction are higher in comparison to $X$ and $Z$ direction. Therefore, 
presented results reveal anisotropy of thermal properties of investigated crystals. Growing dopants concentration causes decrease in the thermal diffusivity. Additionally, it also reduces differences between the thermal diffusivities determined for different directions in the crystal. Dopants can reduce crystal anisotropy.

\section{Conclusions}

A few GdCOB single crystals with different doping concentration grown by the Czochralski method were investigated to find their thermal properties. The thermal diffusivity was determined from thermal wave measurements with a mirage detection. The obtained results showed anisotropy of thermal properties. According to monoclinic symmetry of the crystal values measured in main optical axes differed from each other. Introduction of $\mathrm{Yb}$ and $\mathrm{Nd}$ ions also changed the thermal diffusivity. This value decreased with increase in doping concentration in the sample. This fact allowed to draw the conclusion that impurity atoms create scattering centers in a crystal structure which results in shortening of phonon mean free paths and lowering of the thermal conductivity.

\section{Acknowledgments}

The current investigation is a part of a research project 3 T08A 03529 .

\section{References}

[1] S. Zhang, Z. Cheng, G. Zhou, Y. Sun, X. Hou, X. Liu, J. Han, Z. Shao, H. Chen, Prog. Cryst. Growth Ch. 40, 81 (2000).

[2] H. Nakaoa, M. Nishida, T. Shikida, H. Shimizu, H. Takeda, T. Shiosaki, J. Alloy Comp. 408-412, 582 (2006).

[3] S. Zhang, Z. Cheng, J. Liu, J. Han, Z. Shao, H. Chen, J. Cryst. Growth 212, 476 (2000).

[4] G. Aka, F. Mougel, F. Augé, A. Kahn-Harari, D. Vivien, J.M. Bénitez, F. Salin, D. Pelenc, F. Balembois, P. Georges, A. Brun, N. Le Nain, M. Jacquet, J. Alloy Comp. 303-304, 401 (2000).

[5] E. Talik, M. Kruczek, A. Pajączkowska, A. Kłos, L. Lipińska, E. Łojko, J. Alloy Comp. 442, 282 (2007).

[6] A. Brenier, A. Majchrowski, E. Michalski, T. Łukasiewicz, Opt. Commun. 217, 395 (2003).

[7] F. Moguel, A. Kahn-Harari, G. Aka, D. Pelenc, J. Mater. Chem. 8, 1619 (1998).

[8] Ch. Wang, H. Zhang, X. Meng, L. Zhu, Y.T. Chow, X. Liu, R. Cheng, Z. Yang, S. Zhang, L. Sun, J. Cryst. Growth 220, 114 (2000).

[9] T. Łukasiewicz, I.V. Kityk, M. Makowska-Janusik, A. Majchrowski, Z. Gałązka, H. Kaddouri, Z. Mierczyk, J. Cryst. Growth 237-239, 641 (2002).

[10] K.L. Muratikov, A.L. Glazov, H.G. Walther, High Temp.-High Press. 31, 69 (1999).

[11] M. Martrou, F. Moguel, G. Aka, A. Kahn-Harari, D. Vivien, B. Viana, OSA, Advanced Solid State Lasers 158, AMF1-1 (1998).

[12] J. Bodzenta, M. Pyka, J. Phys. IV (France) 137, 259 (2006). 